\title{
Why Value Framework Assessments Arrive at Different Conclusions: A Multiple Myeloma Case Study
}

\author{
Kimberly Westrich, MA; Lisabeth Buelt, MPH; and Robert W. Dubois, MD, PhD
}

\begin{abstract}
SUMMARY
As the United States transitions from a volume-based health care system to one that rewards value, new frameworks are emerging to help patients, providers, and payers assess the value of medical services and biopharmaceutical products. These value assessment frameworks are intended to support various types of health care decision making. They have the potential to substantially affect patients, whether as tools for shared decision making with their doctors, as an input to care pathways used by providers, or through payer use of the frameworks to make coverage or reimbursement decisions.

Prominent among current U.S. value assessment frameworks are those developed by the American Society of Clinical Oncology, the Institute for Clinical and Economic Review, the Memorial Sloan Kettering Cancer Center, and the National Comprehensive Cancer Network. These frameworks generally reflect the interests and expertise of the organizations that developed them.

The evidence, methodology, and intended use differ substantially across frameworks, which can lead to highly variable determinations of value for the same treatment therapy. To demonstrate this variability, we explored how these frameworks assess the value of treatment regimens for multiple myeloma. Cross-framework comparisons of multiple myeloma assessments were conducted, and consistency of findings was examined for 3 case studies. A discussion of the analysis explores why different frameworks arrive at different conclusions, whether those differences are cause for concern, and the resulting implications for framework readiness to support health care decision making.
\end{abstract}

J Manag Care Spec Pharm. 2017;23(6-a):S28-S33

Copyright $\odot 2017$, Academy of Managed Care Pharmacy. All rights reserved.

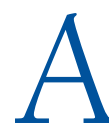
s the United States transitions from a volume-based health care system to one that rewards value, new frameworks are emerging to help stakeholders assess the value of medical treatments. These value assessment frameworks are intended to support various types of health care decision making, and they have the potential to substantially affect patients, whether as tools for shared decision making with their doctors, as an input to care pathways used by providers, or through payer use of the frameworks to make coverage or reimbursement decisions.

Prominent among current U.S. value assessment frameworks are frameworks developed by the American Society of Clinical Oncology (ASCO), the Institute for Clinical and Economic Review (ICER), the Memorial Sloan Kettering Cancer Center (DrugAbacus), and the National Comprehensive Cancer Network (NCCN). Each of these groups has assessed the value of various multiple myeloma treatment regimens. Although one might expect the various assessments to yield similar answers, our review of the assessments finds that this expectation is not realized. This article considers why different frameworks arrive at different findings, whether those differences are cause for concern, and the resulting implications for framework readiness to support health care decision making.

\section{Framework Overview}

The common goal of framework developers is the assessment of the value of a treatment regimen in order to inform health care decision making. The value of a treatment regimen includes some measure of the benefits received and the cost of receiving those benefits. Each of the frameworks focused on here have different purposes and audiences, as well as different approaches to defining and measuring benefits and costs.

\section{American Society of Clinical Oncology}

The ASCO Conceptual Framework to Assess the Value of Cancer Treatment Options is a shared decision-making tool for patients and providers. ${ }^{1}$ In its current version, the tool uses data from a single randomized controlled trial to produce a numerical estimate of net health benefit (NHB) for a treatment regimen relative to a comparator regimen. Points are assigned for clinical benefit, toxicity, and bonus items such as improved quality of life. The summation of points produces an NHB score. The framework also reports the drug acquisition cost for the regimens. ASCO has released 2 versions of its framework and is in the process of developing a software tool. This analysis uses framework version 1.0, the version used by ASCO for their multiple myeloma assessment.

\section{National Comprehensive Cancer Network}

The NCCN Evidence Blocks is also a shared decision-making tool for patients and providers, and the assessments are embedded in the NCCN clinical guidelines. ${ }^{2}$ To evaluate a regimen, panel members consider the body of evidence in NCCN guidelines and their clinical experience to assign a score of 1 to 5 for each of 5 categories: efficacy, safety, quality of evidence, consistency of evidence, and affordability. For each treatment regimen, scores are averaged across panel members to produce a single score for each of the 5 categories. NCCN guidelines are regularly updated. This analysis used version 3.16 of the multiple myeloma guidelines. 
TABLE 1 Comparison of Regimens Examined by Selected Frameworks

\begin{tabular}{|c|c|c|c|}
\hline Domain & ASCO & NCCN & Comparison of Framework Assessments \\
\hline Efficacy & $\begin{array}{l}47 \text { points for treatment (relative to } \\
\text { comparator) }\end{array}$ & $\begin{array}{l}\text { - Treatment: } 4 \text { (very effective) } \\
\text { - Comparator: } 3 \text { (moderately effective) }\end{array}$ & $\begin{array}{l}\text { Both: treatment more efficacious than } \\
\text { comparator }\end{array}$ \\
\hline Toxicity & $\begin{array}{l}0 \text { points for treatment (relative to } \\
\text { comparator) }\end{array}$ & $\begin{array}{l}\text { - Treatment: } 3 \text { (mildly toxic) } \\
\text { - Comparator: } 4 \text { (occasionally toxic) }\end{array}$ & $\begin{array}{l}\text { - ASCO: no toxicity difference between the } \\
2 \text { regimens } \\
\text { - NCCN: treatment regimen more toxic than } \\
\text { comparator }\end{array}$ \\
\hline Economic & $\begin{array}{l}\text { - Treatment: } \$ 7,042 / \text { month } \\
\text { - Comparator: } \$ 279 / \text { month }\end{array}$ & $\begin{array}{l}\text { - Treatment: } 3 \text { (modestly expensive) } \\
\text { - Comparator: } 4 \text { (inexpensive) }\end{array}$ & $\begin{array}{l}\text { Both: treatment more expensive than } \\
\text { comparator }\end{array}$ \\
\hline \multicolumn{4}{|c|}{ Case Study \#2: Comparison of DrugAbacus and NCCN Assessments for BOR (Treatment) } \\
\hline Domain & DrugAbacus & NCCN & Comparison of Framework Assessments \\
\hline Efficacy & Not directly observable & 3 (moderately effective) & $\begin{array}{l}\text { - DrugAbacus: efficacy unknown } \\
\text { - NCCN: moderately effective }\end{array}$ \\
\hline Toxicity & Not directly observable & 4 (occasionally toxic) & $\begin{array}{l}\text { - DrugAbacus: toxicity unknown } \\
\text { - NCCN: occasionally toxic }\end{array}$ \\
\hline Economic & $\begin{array}{l}\text { - DrugAbacus estimated value-based price } \\
\text { range: } \$ 841-\$ 728,361 \\
\text { - Actual monthly cost to Medicare: } \$ 4,474\end{array}$ & 2 (expensive) & $\begin{array}{l}\text { - DrugAbacus: wide range of value-based } \\
\text { prices } \\
\text { - NCCN: expensive }\end{array}$ \\
\hline \multicolumn{4}{|c|}{ Case Study \#3: Comparison of ICER and NCCN Assessments for PAN + BOR + DEX (Treatment) Relative to BOR + DEX (Comparator) } \\
\hline Domain & ICER & $\mathrm{NCCN}$ & Comparison of Framework Assessments \\
\hline Efficacy & $\begin{array}{l}\text { - OS: no difference between treatment and } \\
\text { comparator } \\
\text { - PFS: treatment superior to comparator }\end{array}$ & $\begin{array}{l}\text { - Treatment: } 3 \text { (moderately effective) } \\
\text { - Comparator: } 4 \text { (very effective) }\end{array}$ & $\begin{array}{l}\text { - ICER: treatment more efficacious than com- } \\
\text { parator (PFS)/no difference between treat- } \\
\text { ment and comparator (OS) } \\
\text { - NCCN: treatment less efficacious than } \\
\text { comparator }\end{array}$ \\
\hline Toxicity & $\begin{array}{l}\text { Treatment has a more severe toxicity profile } \\
\text { than comparator }\end{array}$ & $\begin{array}{l}\text { - Treatment: } 2 \text { (moderately toxic) } \\
\text { - Comparator: } 4 \text { (occasionally toxic) }\end{array}$ & Both: treatment more toxic than comparator \\
\hline Evidence & $\begin{array}{l}\text { Insufficient evidence available to evaluate } \\
\text { treatment for second-line therapy; promising, } \\
\text { but inconclusive, evidence available to } \\
\text { evaluate treatment for third-line therapy }\end{array}$ & $\begin{array}{l}\text { Evidence quality } \\
\text { - Treatment: } 4 \text { (good quality evidence) } \\
\text { - Comparator: } 4 \text { (good quality evidence) } \\
\text { Evidence consistency } \\
\text { - Treatment: } 4 \text { (evidence mainly consistent) } \\
\text { - Comparator: } 4 \text { (evidence mainly consistent) }\end{array}$ & $\begin{array}{l}\text { - ICER: evidence is insufficient/inconclusive } \\
\text { to evaluate treatment } \\
\text { - NCCN: evidence is of good quality and } \\
\text { mainly consistent for evaluation of treat- } \\
\text { ment and comparator }\end{array}$ \\
\hline Economic & $\begin{array}{l}\text { - Treatment is cost-effective } \\
\text { - No price discount needed }\end{array}$ & Both regimens: 2 (expensive) & $\begin{array}{l}\text { - ICER: treatment cost-effective, no discount } \\
\text { needed } \\
\text { - NCCN: no cost difference between regimens }\end{array}$ \\
\hline
\end{tabular}

$A S C O=$ American Society of Clinical Oncology; $B O R=$ bortezomib; $D E X=$ dexamethasone; ICER = Institute for Clinical and Economic Review; $M E L=$ melphalan; $N C C N=$ National Comprehensive Cancer Network; OS = overall survival; $P A N=$ panobinostat; $P F S=$ progression-free survival; $P R E D=$ prednisone.

\section{DrugAbacus}

DrugAbacus is an online web tool primarily aimed at policymakers and payers. ${ }^{3}$ It is designed to explore value-based prices for oncology drugs based on 8 components: efficacy, toxicity, novelty, development costs, rarity, population burden, unmet need, and disease prognosis. The values assessed by DrugAbacus for these components are embedded in the web tool and are not visible to the user. Efficacy and toxicity data come from clinical trial results used to gain approval by the U.S. Food and Drug Administration. The user of the tool can select different weights for each of the components, and the tool uses those weights to generate a value-based price.

\section{Institute for Clinical and Economic Review}

ICER's value assessment framework is designed to provide assessments of value to inform payer coverage and reimbursement decisions. ${ }^{4}$ To evaluate a treatment regimen, ICER models the comparative clinical effectiveness and calculates the incremental cost to achieve a unit of clinical outcome (e.g., cost per quality-adjusted life-year gained). ICER identifies other considerations (e.g., improved productivity at work) in a qualitative 


\begin{tabular}{|c|c|c|}
\hline \multicolumn{3}{|c|}{ Case Study \#1: Generally Consistent } \\
\hline Domain & Consistency & Explanation for Inconsistency \\
\hline Efficacy & Yes & N/A \\
\hline Toxicity & No & Measuring different types of toxicities \\
\hline Economic & Yes & N/A \\
\hline \multicolumn{3}{|c|}{ Case Study \#2: Indeterminate Consistency } \\
\hline Domain & Consistency & Explanation for Inconsistency \\
\hline Efficacy & Unknown & DrugAbacus estimate unobservable \\
\hline Toxicity & Unknown & DrugAbacus estimate unobservable \\
\hline Economic & Unknown & $\begin{array}{l}\text { Unclear how to interpret DrugAbacus } \\
\text { estimated prices }\end{array}$ \\
\hline \multicolumn{3}{|c|}{ Case Study \#3: Generally Inconsistent } \\
\hline Domain & Consistency & Explanation for Inconsistency \\
\hline Efficacy & No & Different evidence bases? \\
\hline Toxicity & Yes & N/A \\
\hline Evidence & No & Different evidence bases? \\
\hline Economic & Unknown & Measuring different economic concepts \\
\hline
\end{tabular}

manner. ICER will release a revised version of its framework in 2017.

\section{Cross-Framework Comparisons of} Multiple Myeloma Assessments

This analysis builds on a previous case study analysis by the Lewin Group, which conducted cross-framework comparisons of multiple myeloma treatment regimens that were assessed by at least 2 value assessment frameworks. An in-depth description of their methods and the case studies -3 of which are used in this analysis-is presented in their analysis. ${ }^{5}$

The goal of this analysis is to identify why framework assessments arrive at inconsistent, sometimes even conflicting, determinations of value when evaluating the same treatment and indication. We considered a comparison to be "consistent" when both frameworks were directionally the same for a given domain (e.g., both ASCO and NCCN found the treatment to be more efficacious than the comparator). An "inconsistent" comparison was one with directional difference (e.g., ICER found the treatment to be more efficacious than the comparator, and NCCN found the treatment to be less efficacious than the comparator).

The most prominent difference in the framework assessments is the variance in the breadth of regimens that were assessed by each. NCCN provides an assessment for every regimen included in its multiple myeloma clinical guidelines. ${ }^{6}$ These assessments are subdivided into different patient populations and indications (e.g., primary therapy for transplant candidates or therapy for previously treated multiple myeloma). In comparison, ASCO has conducted a pilot assessment for 1 regimen indicated for multiple myeloma not previously treated. ${ }^{7}$ The online tool for DrugAbacus includes 3 drugs indicated for relapsed or refractory multiple myeloma. ${ }^{8}$ ICER assesses 6 regimens indicated for second-line or later treatment of relapsed or refractory multiple myeloma. ${ }^{8}$

Given the different audiences and purposes of the various frameworks, it is not surprising that each one addresses somewhat different sets of questions toward meeting the needs of their different sets of stakeholders, including differences in patient indications, regimens, and approaches to assessing clinical and economic parameters. Although there are broad framing differences in the 4 assessments, our analysis of these 3 Lewin Group case studies found areas of overlap and inconsistency.

\section{Case Study \#1: Generally Consistent}

The ASCO framework and NCCN Evidence Blocks assessed the treatment regimen bortezomib + melphalan + prednisone (BOR + MEL + PRED) for active multiple myeloma not previously treated. Since the ASCO framework assessment compared BOR + MEL + PRED with the control regimen MEL+ PRED, we considered the NCCN Evidence Blocks for both of these regimens as part of this case study (Table 1). We found 2 areas of consistency and 1 area of inconsistency (Table 2).

Efficacy. Both assessments found that the treatment regimen was more efficacious than the comparator. ASCO awarded 47 NHB points to the treatment regimen relative to the comparator. NCCN rated the treatment regimen 4 (very effective) and the comparator 3 (moderately effective; Table 1).

Toxicity. The toxicity assessments differed. ASCO awarded zero NHB points, suggesting that the treatment toxicity is similar to the comparator, while NCCN rated the treatment regimen as more toxic than the comparator (Table 1). The reason for the inconsistency is likely because of the way toxicity was measured: ASCO focused on the more severe grade 3-5 toxicities, while NCCN evaluated toxicity more broadly (Table 2). Both approaches appear to be reasonable, and this inconsistency is not necessarily a cause for concern. However, it is important for end users to understand the underlying reason for the discrepancy and to consider the different toxicity perspectives when making a treatment choice.

Economic. Both economic assessments suggested that the treatment regimen was more expensive than the comparator. However, a direct comparison of monthly drug acquisition costs (ASCO) with broad categorical affordability labels (NCCN) is not straightforward: Does ASCO's $\$ 7,042$ per month equate to NCCN's "modestly expensive," and does ASCO's $\$ 279$ per month equate to NCCN's "inexpensive" (Table 1)? It is also worth noting that neither of these assessments focused on cost to the patient, which is the most relevant measure given 
each tool's shared decision-making focus. In addition, neither one specifically addressed duration of treatment.

\section{Case Study \#2: Indeterminate Consistency}

DrugAbacus and the NCCN Evidence Blocks assessed BOR as a treatment for relapsed or refractory multiple myeloma. We found 3 areas where we could not determine whether there was agreement or disagreement (Table 2).

Efficacy. The DrugAbacus efficacy estimate was embedded in the web tool and cannot be observed by the user, leaving us unable to determine whether its estimate is consistent with the NCCN score of 3 (moderately effective; Table 1).

Toxicity. As with the efficacy estimate, the DrugAbacus toxicity estimate was not visible to the user, leaving us unable to determine whether its estimate is consistent with the NCCN score of 4 (occasionally toxic; Table 1).

Economic. It was also not possible to determine whether the economic assessments are consistent. NCCN assigned a score of 2 (expensive). DrugAbacus reported that the monthly cost to Medicare was $\$ 4,474$, and changing the preference weights on the tool produced estimated prices ranging from $\$ 841$ to $\$ 728,361$ (Table 1). It is unclear to the user how to interpret this range of potential prices, and whether it is consistent with NCCN's score.

\section{Case Study \#3: Generally Inconsistent}

The NCCN Evidence Blocks and the ICER framework assessed the treatment regimen panobinostat + bortezomib + dexamethasone $(\mathrm{PAN}+\mathrm{BOR}+\mathrm{DEX})$ for relapsed or refractory multiple myeloma. ICER's assessment was relative to the comparator regimen (BOR+DEX), so we considered NCCN's assessments for both regimens as part of this case study. We found 1 area of consistency, 2 areas of noteworthy inconsistency, and 1 area of unknown consistency (Table 2).

Efficacy. The efficacy assessments produced conflicting results. ICER found that progression-free survival was higher for the treatment regimen and overall survival was not statistically different between the 2 regimens. In contrast, NCCN scored the treatment regimen as less efficacious than the comparator regimen (Table 1). The reason for the inconsistency is unclear, but it may be caused by differing evidence bases (Table 2). ICER's findings came from specific clinical trials. NCCN likely used a broader body of evidence and clinical experience, but we do not know the precise underlying evidence base or the thought processes that informed the efficacy scores of the NCCN panel members.

Toxicity. Both assessments found that the treatment regimen was more toxic than the comparator. NCCN scored the treatment regimen as 2 (moderately toxic) and the comparator as 4 (occasionally toxic), while ICER found that the treatment regimen presented a more severe toxicity profile relative to other regimens (Table 1).

Evidence Assessment. There is inconsistency between NCCN and ICER's assessments of the evidence base they used. While NCCN scored the evidence as good quality and mainly consistent, ICER rated the available evidence as insufficient for second-line therapy and promising, but inconclusive, for third-line subsequent therapy (Table 1). Again, the reason for the inconsistency is unclear but may be a result of differing evidence bases (Table 2).

Economic. Economic assessments by ICER and NCCN cannot be compared for consistency because they measure different things. The ICER framework found that the treatment was cost-effective and that no price discount was needed, whereas NCCN assigned an affordability score of 2 (expensive; Table 2).

\section{Discussion}

Although one might expect the various multiple myeloma value assessments to arrive at generally consistent results, we found that consistency varied across the 3 case studies. It is illuminating to consider the reasons why different frameworks might arrive at different, or even incomparable, results and whether these inconsistencies indicate that changes are needed before using assessment results to inform health care decisions.

The inconsistencies observed in case study \#3 are of concern. The efficacy assessments are in direct conflict, as are the evaluations of the quality of the evidence base. Why would these assessments be so different? We know what evidence drives the ICER efficacy assessment. For NCCN, we are unsure of the precise evidence base that the experts had in mind when they were assigning efficacy scores. We know that the body of evidence in the NCCN guidelines and the clinical experiences of the experts were inputs to the scoring process, but we do not know how those inputs led to the ultimate score that was assigned, and we cannot reproduce it. Similarly, we do not know how those inputs led to the NCCN scores signifying high quality evidence that is mainly consistent. Could problems arise from payers making decisions using ICER's assessments, while patients and their providers make decisions using NCCN's assessments, given the conflicts between the 2 assessments?

When consistency of results cannot be determined, it could be a result of unobservable data, which is cause for concern. In case study \#2, DrugAbacus estimates for efficacy and toxicity are not visible to the user. They may be consistent with the NCCN results, but we do not know because there is no transparency. Without transparency, an assessment cannot be reproduced or verified, and credibility may be called into question.

Another reason why consistency of results may be indeterminate is that assessment inputs rely on completely different 
concepts, as occurs twice with economic assessments. In case study \#2, DrugAbacus produced a wide range of estimated prices, as it is designed to do. Since DrugAbacus does not presume any single right answer, it is not clear what this range means or how the user should interpret it, and it is not possible to compare this economic assessment to the NCCN score of 2 (expensive). In case study \#3, ICER's economic assessment was in the form of a cost-effectiveness analysis. It concluded that the treatment was cost-effective and that no price discount was needed. Again, it is not possible to compare ICER's comparative cost assessment to NCCN's stand-alone affordability score of 2 (expensive).

This lack of comparability is not necessarily a concern, but it could contribute to misinterpretation or misuse of the value assessments. If the economic assessments are fit for purpose and usable by the intended audience, the different approaches may make sense. ICER's primary audience is payers, who may be seeking cost-effectiveness information and a basis for seeking price discounts. NCCN's primary goal is shared decision making, and patients may be interested in a different type of economic information (e.g., how much is my copay?). However, when comparability is not possible, we cannot determine if there is conflicting information, which may lead to conflicting health care decisions, as appears to be the case between the ICER and NCCN assessments in case study \#3.

Only a third of the assessment results were clearly consistent across the 3 case studies. At best, lack of consistency can lead to confusion among end users. At worst, it could lead to misinformed or conflicting health care decisions. Both cases suggest that these tools are not ready for widespread adoption and use for the purposes of policy development and clinical shared decision making.

\section{Opportunities for Improving Quality and Utility of Frameworks}

The underlying reasons for lack of consistency point to some opportunities for improvement in the areas of transparency and usability.

1. Improve transparency. Transparency affects the reproducibility and interpretation of assessment results. To enhance the accessibility and credibility of value framework assessments, full transparency is needed for the following: (a) the specific body of evidence underlying an assessment; (b) how various pieces of that evidence drove an assessment's results; (c) the process by which the evidence was transformed into components of the assessment; and (d) the actual values of those components.

2. Enhance usability. Usability of the frameworks can be improved by ensuring that assessment results are fit for purpose and provide more information on how to interpret and use the results. For example, a fit-for-purpose economic assessment for shared decision making would provide information about costs to the patient. Information on interpreting results is particularly important in situations where assessments are creating new and unfamiliar measures, such as the ASCO NHB score and the DrugAbacus preference-weighted range of prices. Guidance on using the results should also extend to who the appropriate users are, since payer-focused assessments may be misleading for patients and vice versa.

\section{Conclusions}

As value becomes increasingly central to health care decision making, value assessment frameworks have the potential to substantially affect stakeholders across the health care continuum, particularly patients. Our analysis of the multiple myeloma framework assessments found several unexplained and indeterminate inconsistencies, which point to clear opportunities for framework developers to deliver assessments that are more transparent, reproducible, and relevant for their respective target users. Considerable inconsistency in value determinations across frameworks could signify that these assessments require further refinement before widespread adoption and use for health care decision making.

\section{Authors}

KIMBERLY WESTRICH, MA; LISABETH BUELT, MPH; and ROBERT W. DUBOIS, MD, PhD, National Pharmaceutical Council, Washington, DC.

AUTHOR CORRESPONDENCE: Kimberly Westrich, MA, 1717 Pennsylvania Ave., NW, Ste. 800, Washington, DC, 20006 Tel.: 202.827.2085; E-mail: kwestrich@npcnow.org.

\section{DISCLOSURES}

Funding for this project was provided by the National Pharmaceutical Council. The authors are employees of the National Pharmaceutical Council, an industry-funded health policy research group that is not involved in lobbying or advocacy.

Study concept and design were contributed by Westrich and Dubois, along with Buelt. Westrich took the lead in data collection, along with Dubois, and data interpretation was performed by all the authors. The manuscript was written by Westrich and Buelt, along with Dubois, and revised by all the authors.

\section{ACKNOWLEDGMENTS}

The authors thank Clifford Goodman, PhD, and Christel Villarivera, MS, of the Lewin Group for their thoughtful review of this manuscript.

\section{REFERENCES}

1. Schnipper LE, Davidson NE, Wollins DS, et al. Updating the American Society of Clinical Oncology value framework: revisions and reflections in response to comments received. J Clin Oncol. 2016;34(24):2925-34. 
2. National Comprehensive Cancer Network. NCCN Clinical Practice Guidelines in Oncology (NCCN Guidelines) with NCCN Evidence Blocks. Available at: http://www.nccn.org/evidenceblocks/. Accessed April 4, 2017.

3. Memorial Sloan Kettering Cancer Center. DrugAbacus FAQs. Available at: http://www.drugabacus.org/drug-abacus/faq/. Accessed April 4, 2017.

4. Institute for Clinical and Economic Review. ICER value assessment framework. Available at: https://icer-review.org/methodology/icers-methods/icervalue-assessment-framework/. Accessed April 4, 2017.

5. The Lewin Group. Comparison of value framework assessments for multiple myeloma. November 2016. Available at: http://www.npcnow.org/system/ files/research/download/170215\%20Lewin\%20Multiple\%20Myeloma\%20 White\%20Paper.pdf. Accessed April 4, 2017.
6. NCCN Evidence Blocks Version 3. Multiple myeloma. 2016. Ft. Washington, PA: National Comprehensive Cancer Network; April 2016.

7. Schnipper LE, Davidson NE, et al. American Society of Clinical Oncology statement: a conceptual framework to assess the value of cancer treatment options. J Clin Oncol. 2015;33(23):2563-77.

8. Ollendorf, D, Chapman, R, Khan, S, et al. Treatment options for relapsed or refractory multiple myeloma: effectiveness, value, and value-based price benchmarks. Final evidence report and meeting summary. June 9, 2016. Available at: https://icer-review.org/wp-content/uploads/2016/06/MWCEPAC_ MM_Final_Evidence_Report_061016V3.pdf. Accessed April 4, 2017. 